\title{
Lognormal Distribution of Cellular Uptake of Radiopharmaceuticals: Implications for Biologic Response in Cancer Treatment
}

$\mathbf{P}$ incorporation of radioactivity largely relies on calculating the absorbed dose. Traditionally, dosimetry has been performed using the mean absorbed dose at the organ level (1) and, more recently, at the suborgan and voxel levels (2). However, even if radioactivity appears to be homogeneous at the voxel level, radiopharmaceuticals do not distribute uniformly at the cellular level (3), either among different cells or among different subcellular compartments (4). This heterogeneous distribution at the cellular level can cause a wide range of

\section{See page 642}

cellular absorbed doses, thus jeopardizing the effectiveness of targeted cancer radiotherapy, especially when radionuclides emitting short-range radiation are used-such as $\alpha$-particles and Auger electrons (5).

One possible strategy to compensate for such heterogeneity in cellular radioactivity incorporation would be the use of isotopes emitting long-range electrons. Labeled cells would thus deliver a cross-dose to nonlabeled cells over longer distances. However, the effectiveness of the cross-dose is hindered by 2 kinds of problems. First, histologic analyses show that the architecture of

Received Dec. 18, 2010; revision accepted Dec. 28, 2010.

For correspondence or reprints contact: Paolo Zanotti-Fregonara, Molecular Imaging Branch, National Institute of Mental Health, Building 10, Room B1D43K6, 10 Center Dr., MSC 2035, Bethesda, MD 20892-2035.

E-mail: zanottifregonp@mail.nih.gov

COPYRIGHT ( 2011 by the Society of Nuclear

Medicine, Inc.

DOI: 10.2967/jnumed.110.084590 tumoral tissues is often heterogeneous and composed of individual tumoral cellular clusters separated by areas of infiltrating inflammatory cells, connective tissue, fibrous septa, and necrotic areas. Therefore, even when using energetic particles such as ${ }^{131} \mathrm{I} \beta$-electrons, completely unlabeled clusters are likely to receive small doses (6). Second, the cross-dose is less lethal per unit dose than is self-dose. In 2004, Neti and Howell determined the in vitro effect of self-dose and cross-dose on ${ }^{131}$ I-iododeoxyuridine-labeled cells (which received both self- and cross-doses), as well as on unlabeled cells (which received only cross-dose). They found that the self-dose was about 3.3 times more radiotoxic per unit dose than the cross-dose (7). The higher relative biological effectiveness of self-dose was likely due to the highly localized energy deposit of low-energy electrons emitted within DNA during ${ }^{131} \mathrm{I}$ decay (7).

These data point to the fact that the mean absorbed dose calculated at the macroscopic level is often inadequate for predicting biologic response. In another elegant experiment, Neti and Howell assembled a 3-dimensional multicellular cluster model of V79 Chinese hamster cells in which a known percentage of cells was radiolabeled with ${ }^{131}$ I-iododeoxyuridine and randomly located in the cluster. Thus, the activity distribution was uniform at the macroscopic level, even if it was not uniform at the cellular level. Colony-forming assays showed that when $100 \%$ of cells were labeled, the survival fraction of cells was exponentially dependent on the cluster activity down to $0.1 \%$ survival. In contrast, when $10 \%$ of the cells were labeled, the survival fraction began to saturate at about $1 \%$ survival (8). Moreover, the study found that to achieve the same level of overall kill, it was necessary to use substantially more radioactivity per cell as the percentage of labeled cells decreased (8).

Taken together, these studies suggested that the most effective targeted radiotherapy would thus be one capable of achieving uniform radiopharmaceutical incorporation into each cell. However, in 2006, Neti and Howell provided important experimental evidence that cellular uptake of radioactivity is not uniform (9). They exposed cultured cells to the $\alpha$-emitting ${ }^{210}$ Po-citrate and used $\alpha$-autoradiography to quantify radioactivity incorporation. Despite the fact that all the cells were exposed to the same extracellular concentration of ${ }^{210} \mathrm{Po}-$ citrate, the investigators found that cellular uptake was not homogeneous but followed a lognormal distribution over a wide range of radioactivity concentrations $(9,10)$. Performing theoretic calculations, they also showed that the breadth of the peak of the lognormal distribution (described by the shape parameter $\sigma$ ) had a profound impact on the survival curve; survival fraction was lower as $\sigma$ became smaller $(\sigma \rightarrow 0$ represents the case where all the cells in the population contain the same activity) (9).

In this issue of The Journal of Nuclear Medicine, Akudugu, Neti, and Howell extend their findings on the relationship between cellular lognormal uptake of therapeutic agents and biologic response (11). In this study, Chinese hamster V79 lung fibroblasts were exposed to increasing concentrations of ${ }^{210} \mathrm{Po}$-citrate and 2 anthracycline chemotherapy drugs 
(daunomycin and doxorubicin). The cellular incorporation of these molecules was quantified using flow cytometry to measure the fluorescence intensity per cell. Cell survival was then assessed using colony-forming assays. Corroborating their previous studies $(9,10)$, the investigators demonstrated that the distribution of intracellular agent concentrations follows a lognormal distribution, not only for the radioactive agent but also for the 2 chemotherapy drugs. In the case of ${ }^{210} \mathrm{Po}$-citrate, the distribution was perfectly lognormal and the $\sigma$ value remained constant throughout the range of extracellular concentrations studied. In contrast, daunomycin and doxorubicin displayed departures from lognormality with varying concentrations, and $\sigma$ values decreased with increasing drug concentration. The clonogenic assays showed a monoexponential relationship between ${ }^{210} \mathrm{Po}-$ citrate incorporation and cell survival over 2 logs of cell killing. For both daunomycin and doxorubicin, however, cell survival and cellular drug uptake were related via 2 exponential functions. For these 2 drugs, changes in the value of the lognormal shape parameter as well as changes in the slope of the cellular uptake curves with increasing drug concentration appeared to correlate with changes in the transition from the first to the second component of the exponential survival curve.

These findings are likely to have important practical consequences for targeted radiotherapy. Presently, therapeutic trials in nuclear medicine are generally conducted using a single agent. Because cellular uptake follows a lognormal distribution, some cells would incorporate subtoxic amounts of any therapeutic agent. As a result, these cells may not only survive therapy but also repopulate in a more resistant neoplastic form. For instance, stem-cell populations have been identified in a range of hematopoietic and solid tumors and might represent the cell of origin of these tumors. Cancer stem cells are metabolically dormant and also express high levels of adeno- sine triphosphate-binding cassette transporters, which actively clear drugs from cells (12). Cancer stem cells, in particular, may thus represent a subpopulation of cells likely to incorporate subtoxic amounts of drugs and subsequently prompt cancer repopulation.

The present study by Akudugu, Neti, and Howell has other key therapeutic implications. For instance, it suggests that the in vitro study of drug biologic parameters can be used to design effective therapeutic cocktails, in which each additional drug would be specifically chosen to target those cells that had low uptake of the other drugs. The final goal would be to achieve a net homogeneous drug distribution $(\sigma \rightarrow 0)$, thereby optimizing the effectiveness of the targeted therapy. However, whereas such in vitro research is clearly an important step forward in our understanding of the biologic response of cellular dose distribution, the challenging step of extrapolating such work to in vivo cancerous tissues remains. Even within a given cancerous tissue, multiple cellular clones may coexist, and the total profile of radioactivity incorporation may be considerably more complex than that obtained in vitro from cell lines. In addition, radiopharmaceuticals are rarely used in simple chemical form; rather, they are typically coupled to a carrier molecule (such as peptides or antibodies) that is directed against receptors or surface antigens. The degree of expression of these antigens directly influences cellular uptake of the drugs. Moreover, cellular uptake in vivo is affected by the tumoral microenvironment-for example, tumor vascularity and vascular permeability, presence of necrotic zones, anatomic barriers, and accessibility to common drug administration routes. This tumoral microenvironment would also condition cell resistance to radiation, such as in hypoxic areas. Finally, irradiated cells may prompt biologic responses in nonirradiated cells. This phenomenon, known as the bystander effect, is responsible for a variety of responses whose type and magnitude depend on such factors as radionuclide, percentage of cells labeled, cellular self-dose, and in vivo tissue microenvironment (13).

Despite our limited understanding of the biologic response to radiation of cancerous tissues in vivo, these studies nevertheless yield several practical implications. Notably,

- Whereas a multitude of factors can influence overall tissue response, ultimately the activity uptake within individual cells of the targeted tissue must be understood to enable the construction of a meaningful dose-response relationship (3). Therefore, the use of in vitro techniques to quantify $(9,11)$ and localize (4) cellular drug biodistribution is an essential first step toward a rational and informed choice of therapeutic agents.

- To maximize the effectiveness of targeted internal therapy, well-designed cocktails of agents with complementary distribution profiles that either target different cellular antigens (14) or are aimed at different receptors should be used. Selectively targeting the cellular clones responsible for cancer repopulation will become an important goal of any therapy aimed at eradicating minimal residual disease.

- To achieve maximum radiobiologic effectiveness, the isotope should preferably be internalized and emit shortrange particles with high relative biological effectiveness. Although biologically less effective, the cross-dose may contribute to the irradiation of unlabeled cells and help overcome anatomic barriers (15). Therefore, vectors should preferably be labeled with isotopes emitting particles with different ranges.

- Finally, targeted therapy is probably more effective in the earliest disease stages (16), before homogeneous drug distribution is further jeopardized by the development of different cellular clones and alterations in tumoral microarchitecture.

\section{ACKNOWLEDGMENTS}

We thank Ioline Henter for careful editing of the manuscript. This work was supported by the Intramural Research Program of the National 
Institute of Mental Health, National Institutes of Health, Department of Health and Human Services (IRPNIMH-NIH-DHHS). The opinions here expressed are those of the authors and do not reflect the opinions of the NIH or the U.S. federal government.

\section{Paolo Zanotti-Fregonara}

National Institutes of Health

Bethesda, Maryland

\section{Elif Hindié}

St. Louis Hospital

Paris, France

\section{REFERENCES}

1. Snyder WS, Ford MR, Warner GG, Watson SB. "S" Absorbed Dose per Unit Cumulated Activity for Selected Radionuclides and Organs. Reston, VA: Society of Nuclear Medicine; 1975.

2. Bolch WE, Bouchet LG, Robertson JS, et al. MIRD pamphlet no. 17: the dosimetry of nonuniform activity distributions-radionuclide $S$ values at the voxel level. J Nucl Med. 1999;40 (suppl):11S-36S.

3. Bolch WE. Further explorations of cellular uptake of radioactivity. J Nucl Med. 2008;49:869-870.

4. Chehade F, de Labriolle-Vaylet C, Moins N, et al. Secondary ion mass spectrometry as a tool for investigating radiopharmaceutical distribution at the cellular level: the example of I-BZA and ${ }^{14} \mathrm{C}$ I-BZA. J Nucl Med. 2005;46:1701-1706.

5. Kassis AI. Therapeutic radionuclides: biophysical and radiobiologic principles. Semin Nucl Med. 2008;38:358-366.

6. Zanotti-Fregonara P, Champion C, Marzola MC, et al. Monte Carlo simulation of electron-dose from ${ }^{131}$ I-targeted tumor cells within a heterogeneous tumor. Cancer Biother Radiopharm. In press.

7. Neti PV, Howell RW. Isolating effects of microscopic nonuniform distributions of ${ }^{131} \mathrm{I}$ on labeled and unlabeled cells. J Nucl Med. 2004; 45:1050-1058.

8. Neti PV, Howell RW. When may a nonuniform distribution of ${ }^{131}$ I be considered uniform? An experimental basis for multicellular dosimetry. $J$ Nucl Med. 2003;44:2019-2026.

9. Neti PV, Howell RW. Log normal distribution of cellular uptake of radioactivity: implications for biologic responses to radiopharmaceuticals. $J$ Nucl Med. 2006;47:1049-1058.

10. Neti PV, Howell RW. Lognormal distribution of cellular uptake of radioactivity: statistical analysis of alpha-particle track autoradiography. J Nucl Med. 2008;49:1009-1016.

11. Akudugu JM, Neti PV, Howell RW. Changes in lognormal shape parameter guide design of patient-specific radiochemotherapy cocktails. $J$ Nucl Med. 2011;52: 642-649.

12. Dean M, Fojo T, Bates S. Tumour stem cells and drug resistance. Nat Rev Cancer. 2005;5:275-284.

13. Howell RW, Neti PV, Pinto M, Gerashchenko BI, Narra VR, Azzam EI. Challenges and progress in predicting biological responses to incorporated radioactivity. Radiat Prot Dosimetry. 2006;122: 521-527.

14. Milenic DE, Brady ED, Garmestani K, Albert PS, Abdulla A, Brechbiel MW. Improved efficacy of alpha-particle-targeted radiation therapy: dual targeting of human epidermal growth factor receptor-2 and tumor-associated glycoprotein 72 . Cancer. 2010;116(4, suppl)1059-1066.

15. Zhu X, Palmer MR, Makrigiorgos GM, Kassis AI. Solid-tumor radionuclide therapy dosimetry: new paradigms in view of tumor microenvironment and angiogenesis. Med Phys. 2010;37:2974-2984.

16. Hindie E, Zanotti-Fregonara P, Keller I, et al. Bone metastases of differentiated thyroid cancer: impact of early I-131-based detection on outcome. Endocr Relat Cancer. 2007;14:799-807. 